\title{
Photonic Chern insulators made of gyromagnetic hyperbolic metamaterials
}

\author{
Ruei-Cheng Shiu, ${ }^{1}$ Hsun-Chi Chan, ${ }^{1}$ Hai-Xiao Wang, ${ }^{1,2}$ and Guang-Yu Guo, ${ }^{1,2}$ \\ ${ }^{1}$ Department of Physics and Center for Theoretical Physics, \\ National Taiwan University, Taipei 10617, Taiwan \\ ${ }^{2}$ Physics Division, National Center for Theoretical Sciences, Hsinchu 30013, Taiwan
}

(Dated: November 20, 2019)

\begin{abstract}
Controlling light propagation using artificial photonic crystals and electromagnetic metamaterials is an important topic in the vibrant field of photonics. Notably, chiral edge states on the surface or at the interface of photonic Chern insulators can be used to make reflection-free waveguides. Here, by both theoretical analysis and electromagnetic simulations, we demonstrate that gyromagnetic hyperbolic metamaterials (GHM) are photonic Chern insulators with superior properties. As a novel mechanism, the simultaneous occurrence of the hyperbolic and gyromagnetic effects in these metamaterials is shown to open the large topological band gaps with gap Chern number of one. Importantly, unlike many other photonic Chern insulators, the GHM Chern insulators possess nonradiative chiral edge modes on their surfaces, and thus allow to fabricate unidirectional waveguides without cladding metals which generally incurr considerable Ohmic loss. Furthermore, the photonic edge states in the proposed Chern insulators are robust against disorder on a wide range of length scales, in strong contrast to crystalline topological insulators, and the light flow direction on the surface of the Chern insulators can be easily flipped by switching the direction of an applied magnetic field. Fascinatingly, we find that negative refraction of the topological surface wave occurs at the boundary between the GHMs with the opposite signs of gyromagnetic parameters. Finally, we show that compared with other photonic topological materials such as chiral hyperbolic materials, the present GHM Chern insulators can be much easier to fabricate.
\end{abstract}

\section{INTRODUCTION}

Control over the propagation of light using artificial photonic crystals ${ }^{12}$ and electromagnetic metamaterials ${ }^{3}$ has received enormous attention in recent decades mainly because of its importance for many applications in the vibrant field of photonics. For example, metamaterials such as left-handed media ${ }^{45}$ have shown promising potential for novel technologies $6 / 7$. In recent decades, great progress in this field has been often made by taking advantages of analogies with electronic systems in solid state physics. For example, the concept of a photonic band gap material ${ }^{1 / 2}$, a man-made system with a periodic dielectric function, was inspired by the electronic Bloch states in a crystalline semiconductor.

More recently, there have been growing interests in using topological photonic materials 8 to manipulate the flow of light, again inspired by the recent developments of electronic topological materials 911 . In particular, the electronic quantum anomalous Hall (QAH) phase is a two-dimensional (2D) bulk ferromagnetic insulator with a nonzero Chern number in the presence of spin-orbit coupling (SOC) but in the absence of applied magnetic fields $12 \mid 13$. Its associated metallic chiral edge states in this Chern insulator carry dissipationless unidirectional electric current. Haldane and Raghu recently proposed 14 to construct analogs of this intriguing $\mathrm{QAH}$ in photonic crystals made of time-reversal symmetry (TRS) breaking materials to realize unidirectional optical waveguides. Subsequently, these topological electromagnetic states in a number of gyromagnetic photonic crystals with broken TRS were further proposed $\sqrt{15}$ and observed 22 . Interestingly, photonic analog of electronic quantum spin
Hall effect in 2D topological insulators with TRS ${ }^{910}$ were also observed in bi-anisotropic photonic crystals 26 .

Nevertheless, investigations of photonic unidirectional edge modes have mostly been limited to topological photonic crystals periodic on the scale of the operational wavelength, and this considerably restricts the applications of topological photonic materials. Very recently, Gao et. al ${ }^{29}$ theoretically demonstrated topological photonic phase in chiral hyperbolic metamaterials (CHM) made of continuous TRS media with photonic edge states robust against disorder on all length scales 29 . In hyperbolic metamaterials 30 , which are plasmonic metamaterials, equi-frequency surfaces (EFSs) of transverse electricfield (TE) and transverse magnetic-field (TM) modes are degenerate on the high-symmetry points in the momentum space [see Fig. 1(b)]. When the bi-anisotropic property (chirality) is introduced in hyperbolic metamaterials with TRS, which then become CHMs, the degeneracies are broken due to the coupling between TE and TM modes 26 and consequently, a nontrivial band gap is opened ${ }^{29}$. The nontrivial topology of the CHM results from the nonzero Berry curvature due to the chirality (equivalent to the SOC in electronic topological insulators) and broken spatial inversion symmetry in continuous medium 31 . Furthermore, the design principle was based on the concept of a Floquet topological insulator (FTI) ${ }^{32}$ where by treating the distance $z$ propagated by a waveguide mode as a timelike coordinate, e.g., a honeycomb lattice of helical waveguides behaves like a FTI.

In this work, as a novel mechanism for controlling light flow, we introduce the photonic Chern insulators made of continuous gyromagnetic hyperbolic metamaterials (GHM) with the band gap opened by the TRS- 
breaking gyromagnetic effect [Figs. 1(b) and (c)]. The non-trivial topology is demonstrated by the calculated Berry curvature and nonzero Chern number due to the broken TRS. The unidirectional backscattering-immune non-radiative edge modes at the interface between the GHM and vacuum are uncovered by the finite-element electromagnetic simulations. As in the photonic quantum spin-Hall insulators (QSHI) made of the CHMs the photonic edge states in our Chern insulators are robust against disorder on all length scales. In contrast to the QSHIs ${ }^{29}$, however, the light flow direction on the surface of our Chern insulators can be easily flipped by switching the direction of applied magnetic fields. Furthermore, our Chern insulators made of the GHM can be easily fabricated.

\section{GYROMAGNETIC HYPERBOLIC METAMATERIAL}

We consider a GHM as a hyperbolic metamateria 30 with the gyromagnetic response which is described by the constitutive relation,

$$
\left(\begin{array}{l}
\mathbf{D} \\
\mathbf{B}
\end{array}\right)=\left(\begin{array}{cc}
\varepsilon_{0} \hat{\varepsilon} & 0 \\
0 & \mu_{0} \hat{\mu}
\end{array}\right)\left(\begin{array}{l}
\mathbf{E} \\
\mathbf{H}
\end{array}\right)
$$

where

$$
\hat{\varepsilon}=\left(\begin{array}{ccc}
\epsilon_{x x} & 0 & 0 \\
0 & \epsilon_{y y} & 0 \\
0 & 0 & \epsilon_{z z}
\end{array}\right), \hat{\mu}=\left(\begin{array}{ccc}
\mu_{x x} & -i \gamma & 0 \\
i \gamma & \mu_{y y} & 0 \\
0 & 0 & \mu_{z z}
\end{array}\right)
$$

are the relative permittivity and permeability tensors, respectively, and $\gamma$ is the gyromagnetic parameter, representing the degree of TRS breaking upon application of a magnetic field in the $z$-direction (gyromagnetic effect). For simplicity, let us set $\mu_{x x}=\mu_{y y}=\mu_{z z}=\mu=1$, $\epsilon_{x x}=\epsilon_{y y}=\alpha$ and $\epsilon_{z z}=\beta$ such that EFS dispersion are isotropic in $X-Y$ plane. The propagation behavior in the gyromagnetic hyperbolic metamaterials can be described by the wave equation of the electric field $\mathbf{E}=\left(E_{x}, E_{y}, E_{z}\right)^{T}$ as

$$
\mathbf{k} \times \hat{\mu}_{r}^{-1} \mathbf{k} \times \mathbf{E}+k_{0}^{2} \hat{\epsilon_{r}} \mathbf{E}=0,
$$

where $\mathbf{k}=\left(k_{x}, k_{x}, k_{z}\right)$ is the wave vector, and $k_{0}=\omega / c$ is the wave number in vacuum. The left side of Eq. (3) can be rewritten in the matrix form as

$$
\left(\begin{array}{ccc}
\alpha k_{0}^{2}-\frac{k_{y}^{2}}{\mu}-A k_{z}^{2} & \frac{k_{x} k_{y}}{\mu}+B k_{z}^{2} & A k_{x} k_{z}-B k_{y} k_{z} \\
\frac{k_{x} k_{y}}{\mu}-B k_{z}^{2} & \alpha k_{0}^{2}-\frac{k_{x}^{2}}{\mu}-A k_{z}^{2} & A k_{y} k_{z}+B k_{x} k_{z} \\
A k_{x} k_{z}+B k_{y} k_{z} & A k_{y} k_{z}-B k_{x} k_{z} & \beta k_{0}^{2}-A k_{t}^{2}
\end{array}\right)
$$

where $k_{x}^{2}+k_{y}^{2}=k_{t}^{2}, A=\frac{\mu}{\mu^{2}-\gamma^{2}}, B=\frac{\gamma}{\mu^{2}-\gamma^{2}}$. The nontrivial solutions of $\mathbf{E}=\left(E_{x}, E_{y}, E_{z}\right)^{T}$ exist when the determinant of Eq. 4 equals to zero, resulting in the eigen equation of

$$
\alpha^{2} \beta k_{0}^{2} \mu-(\alpha A \mu+\beta-A) \alpha k_{t}^{2}+(A-2 \alpha \mu A) \beta k_{z}^{2}=0 .
$$

Starting with an isotropic optical medium with $\epsilon_{x x}=$ $\epsilon_{z z}=2$ and $\gamma=0$, one has two routes to arrive at a GHM, as illustrated in Fig. 1. The EFS dispersion of the isotropic medium is a perfect sphere, as shown in Fig. 1(a). Furthermore, the eigenstates consisting of transverse electric mode TE $\left(E_{x}=E_{y}=0, H_{z} \neq 0\right)$ and transverse magnetic modes TM $\left(H_{x}=H_{y}=0, E_{z} \neq 0\right)$, are degenerate and their dispersion are identical and on the same sphere. When the isotropic medium is transformed to a hyperbolic metamaterial $\left(\epsilon_{x x}=2, \epsilon_{z z}=-1\right.$, and $\gamma=0$ ) by varying $\epsilon_{z z}$ from 2 to -1 , the EFS sphere of the TM mode splits and becomes two parabola along the $k_{z}$-axis while that of the TE mode remains spherical, as depicted in Fig. 1(b) [see Fig. 2(a, b) for polarization analysis]. This is because TM "sees" $\epsilon_{x x}=\epsilon_{y y}=2$ only while, in contrast, TM "sees" $\epsilon_{x-y}=2$ and $=\epsilon_{z z}=-1$. Interestingly, the two TM parabola touch the TE sphere at $k_{z}= \pm 1.0 k_{0}$, respectively. When the gyromagnetic effect is further introduced into the system by making $\gamma$ nonzero (e.g., $\gamma=0.8$ ), the system becomes a GHM. As a result, a band gap is opened at both degenerate points $k_{z}= \pm 1.0 k_{0}$ [see Fig. 1(d)]. Interestingly, since the gyromagnetic effect breaks TRS, the eigenstates near $\pm 1.0 k_{0}$ become circularly (elliptically) polarized, while eigenstates on the upper and lower hyperbolic bands far away from the singular points remain TM-polarized [see Fig. 2(c,d)].

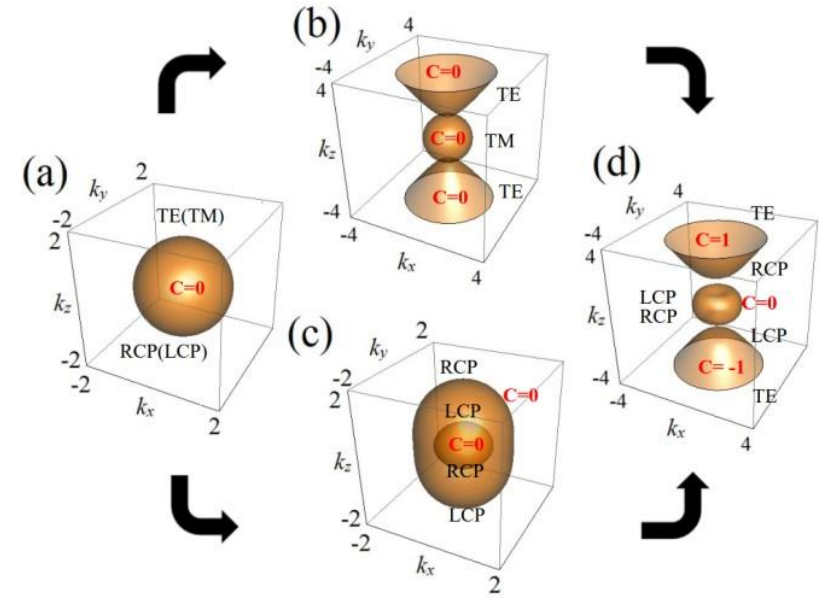

FIG. 1. EFS evolution from (a) an isotropic medium $\left(\epsilon_{x x}=\right.$ $\epsilon_{z z}=2$ and $\left.\gamma=0\right)$ to (d) a GHM $\left(\epsilon_{x x}=2, \epsilon_{z z}=-1\right.$, and $\gamma=0.8)$ via either (b) a hyperbolic metamaterial $\left(\epsilon_{x x}=\right.$ $2, \epsilon_{z z}=-1$, and $\gamma=0$ ) or (c) a gyromagnetic medium $\left(\epsilon_{x x}=\right.$ $\epsilon_{z z}=2$ and $\gamma=0.8$ ). TE (TM) and RCP (LCP) denote TE (TM) polarization and right-handed (left-handed) circular polarization, respectively.

Alternatively, one can go from an isotropic medium first to a gyromagnetic medium (e.g., $\epsilon_{x x}=\epsilon_{z z}=2$ and $\gamma=0.8)$ [see Fig. 1(c)] and then to the GHM. In a gyromagnetic medium, because of broken TRS, TE and TM modes are coupled, resulting in the separation of the degenerate EFS spheres into two ellipsoids [Fig. 1(c)]. Also eigenstates becomes elliptically polarized [see Fig. 
$2(e, f)]$. In particular, the eigenstates on the top and bottom surfaces of the ellipsoids become nearly fully circularly polarized, while those in the vicinity of the $k_{z}=0$ plane remain almost purely either (inner ellipsoid) TE or (outer ellipsoid) TM polarization. Finally, when $\epsilon_{z z}$ is tunned to a negative value (e.g. $\epsilon_{z z}=-1$ ), the system becomes a GHM and the outer ellisoid splits to form two open parabolas, as shown in Fig. 1(d).
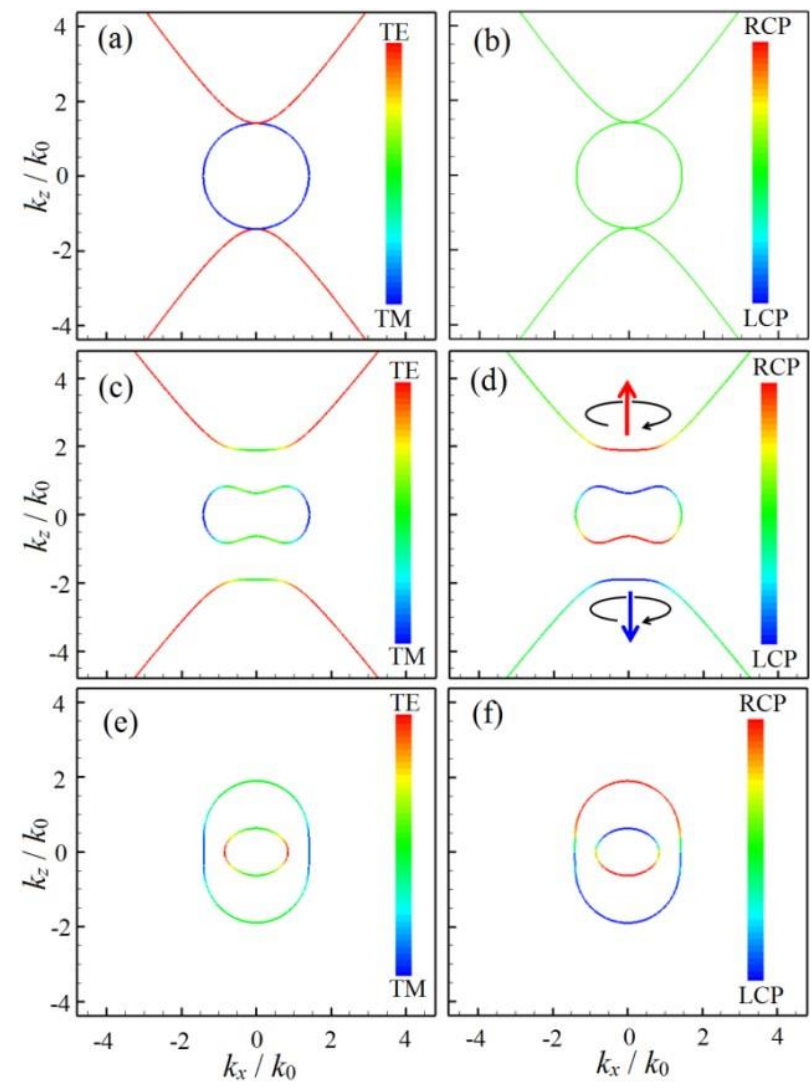

(f)

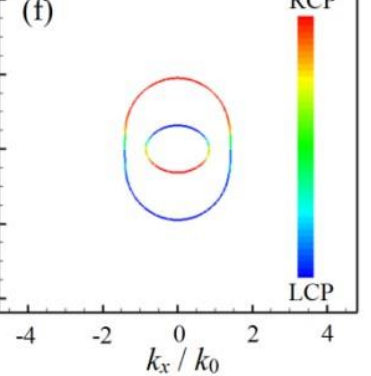

FIG. 2. Polarization analysis of eigenstates. Calculated polarization of EFS dispersion for $(a, b)$ the hyperbolic metamaterial, (c, d) the GHM (c, d) and (e, f) the gyromagnetic medium. RCP (LCP) and TE (TM) denote right-handed (left-handed) circular polarization and TE (TM) polarization, respectively.

\section{TOPOLOGICAL PHASE TRANSITION}

To investigate the topological property of the EFS dispersions of all four kinds of optical media and metamaterials and also to examine the topological nature of the band gaps near $k_{z}= \pm k_{0}$, we calculate the Berry phase $\Phi=\iint \mathbf{F}(\mathbf{k}) \cdot d s$ and hence the Chern number $\mathcal{C}=\frac{1}{2 \pi} \Phi$ of all EFS surfaces. ${ }^{2133}$ Following our previous work ${ }^{21}$, we adopt the efficient numerical algorithm reported in Ref. 33] to evaluate the Berry curvature $\mathbf{F}(\mathbf{k})$. Note that in principle, the Berry phase and the Chern number are well defined only for a closed surface. Fortu- nately, our test calculations show that the Berry curvature $\mathbf{F}(\mathbf{k})$ is negligibly small when the radial wave vector $k_{\rho}=\sqrt{k_{x}^{2}+k_{y}^{2}}>15$. Therefore, although the hyperbolic shaped EFS surfaces are open (Figs. 1 and 2), we find that the calculated Chern numbers would converge well to integers as long as the surface integration is carried out from the $k_{0}$ up to the radial wave vector $k_{\rho}=\sqrt{k_{x}^{2}+k_{y}^{2}}$ being larger than 15 . The Chern numbers calculated in this way are shown in Fig. 1, and the Berry curvature distributions are displayed in Fig. 3. We notice that the Berry curvature has the following symmetry properties: (a) $\mathbf{F}(\mathbf{k})=\mathbf{F}(-\mathbf{k})$ if the system has the spatial inversion symmetry (IS) and (b) $\mathbf{F}(\mathbf{k})=-\mathbf{F}(-\mathbf{k})$ if the system has the TRS symmetry. Therefore, the Berry curvature is identically zero and hence the Chern number is zero for both isotropic medium and hyperbolic metamaterial because they have both IS and TRS [See Figs. 1(a) and $1(\mathrm{~b})]$.


FIG. 3. Berry curvature distribution on EFSs for the gyromagnetic hyperbolic metamaterials with (a) $\alpha=\beta=2$, and $\gamma=0.8$, (b) $\alpha=2, \beta=-1$, and $\gamma=1.2$.

In contrast, the Berry curvatures become nonzero in both gyromagnetic medium and GHM since their TRS is broken, as shown in Fig. 3. Nevertheless, in the gyromagnetic medium, the Berry curvatures of the eigenstates on each EFS ellipsoid form an odd function of $k_{z}$ with respect to the out-surface element [Fig. 3(a)]. Consequently, the sum of the Berry curvatures on the EFS surface of each ellipsoid (i.e., the Berry phase and the Chern number) remains zero [Fig. 1(c)]. This is also true for the inner ellipsoid in the GHM [Fig. 3(b)] and hence the Chern number of the inner EFS is zero [Fig. 1(d)]. However, the outer ellipsoid in the GHM now splits and hence transforms into two open parabolic surfaces with the Berry curvatures pointing to the positive $z$-direction. Although the Berry curvatures on the upper and lower surfaces still form an odd function of $k_{z}$, they are on the different surfaces. Resultantly, the upper and lower open surfaces each acquires a Chern number of -1 . Therefore, the phase transformation from the hyperbolic to gyromagnetic hyperbolic due to the gyromagnetic effect is a topological one, and the band gaps centered at $\pm k_{0}$ are 
topological nontrivial.

\section{NONRADIATIVE ONE-WAY PHOTONIC EDGE MODE}

Next, we follow the method suggested in Ref. 34 to calculate analytically the surface band between the di- electric and anisotropic material. Let the interface between air $(y>0)$ and GHM $(y<0)$ be on the $x z$ plane $(y=0)$. The wave vector in GHM normal to the interface can then be obtained by solving Eq. (5)

$$
k_{y}^{G H M}= \pm\left(\frac{\alpha k_{0}^{2}\left(\alpha \mu^{2}+\beta\left(\mu^{2}-\kappa^{2}\right)\right)-2 \alpha \mu k_{x}^{2}-\alpha \mu k_{z}^{2}-\beta \mu k_{z}^{2} \pm Y}{2 \alpha \mu}\right)^{0.5}
$$

where

$$
Y \equiv \sqrt{\alpha^{2} k_{0}^{4}\left(\alpha \mu^{2}+\beta\left(\kappa^{2}-\mu^{2}\right)\right)^{2}+2 \alpha k_{0}^{2} \mu k_{z}^{2}\left(-\alpha^{2} \mu^{2}+\alpha \beta\left(\kappa^{2}+2 \mu^{2}\right)+\beta^{2}\left(\kappa^{2}-\mu^{2}\right)\right)+\mu^{2}(\alpha-\beta)^{2} k_{z}^{4}}
$$

Then two linear independent eigen fields in GHM $\vec{E}_{i}\left(k_{x}, k_{y}, k_{z}\right)(i=3,4)$ can be obtained by solving the null space of Eq. (4) with $k_{y}=k_{y}\left(k_{x}, k_{z}\right)$ from Eq. (6). Once $\vec{E}_{i}$ are obtained, $\vec{H}_{i}$ can also be obtained via Faraday's law $\vec{H}_{i}=\frac{1}{Z_{0}} \vec{k} \times \vec{E}_{i}$. Likewise, two orthogonal eigen fields in the air can be expressed as

$$
\vec{E}_{1}=\left(\begin{array}{c}
-k_{z} \\
0 \\
k_{x}
\end{array}\right), \quad \vec{E}_{2}=\left(\begin{array}{c}
-k_{y} \\
k_{x} \\
0
\end{array}\right), \quad \vec{H}_{1}=\frac{1}{Z_{0}}\left(\begin{array}{c}
k_{x} k_{y} \\
-k_{x}^{2}-k_{z}^{2} \\
k_{y} k_{z}
\end{array}\right), \quad \vec{H}_{2}=\frac{1}{Z_{0}}\left(\begin{array}{c}
-k_{x} k_{z} \\
-k_{y} k_{z} \\
k_{x}^{2}+k_{y}^{2}
\end{array}\right)
$$

where $Z_{0}=\sqrt{\mu_{0} / \epsilon_{0}}$ is the vacuum impedance, and $k_{y}=i \sqrt{k_{x}^{2}+k_{z}^{2}-k_{0}^{2}}$. Since the tangential components of electromagnetic fields should be continuous across the interface, we arrive at $\operatorname{Det}(D)=0$ with

$$
D\left(k_{x}, k_{z}\right)=\left(\begin{array}{cccc}
-k_{z} & -k_{y} & E_{3 x} & E_{4 x} \\
k_{x} & 0 & E_{3 z} & E_{4 z} \\
k_{x} k_{y} & -k_{x} k_{z} & H_{3 x} & H_{4 x} \\
k_{y} k_{z} & k_{x}^{2}+k_{y}^{2} & H_{3 z} & H_{4 z}
\end{array}\right)
$$

In order to obtain a surface mode, it is important to choose the range of the imaginary part of $k_{y}\left(k_{y}^{G H M}\right)$ in the vacuum (GHM) to be positive (negative). In other words, the fields in the air and GHM must decay exponentially along the vertical direction of the interface.

According to the bulk-edge correspondence, the gap Chern numbers of $\Delta C=-1$ implies that there is one propagating edge state in each band gap for each airGHM interface. Furthermore, these edge states are chiral and topology-protected, i.e., they are reflection-free oneway edge states. To verify these amazing predictions, we solve the Maxwells equations for a slab made of the GHM, as illustrated in Fig. 4(a). The calculated EFS dispersions for this slab, displayed in Fig. 4(b), show that in addition to the bulk bands (black curves), there is indeed one edge state for both top (red curve) and bottom (blue curve) interface between air and the GHM slab in each band gap.

We further simulate light propagation in the air-GHM interface by putting a line source along the $z$-axis at $x=0$. The results are displayed in Figs. 4(c)-4(f). Our simulations show clearly that light on each interface runs without radiation in either $+x$ or $x$ direction but not both directions, i.e., light propagation on the edges is unidirectional. We notice that in Fig. 4(c), both edges at $k_{z}=1.6 k_{0}$ carry light towards $x$ direction. This is because the dispersions of both bulk and edge states are symmetric with respect to the mirror located at $k_{z}=0$. And the propagation along the $x$ direction is caused by the negative gap Chern numbers $(\Delta C=-1)$ of both band gaps. Moreover, Fig. 4(g) shows that light can overcome the obstacles on the surface, indicating light propagation is reflection-free due to the topological protection. Finally, the direction of light propagation depends on the sign of gyromagnetic parameter $\gamma$ [see Figs. 4(c) and $4(d)$, demonstrating that one can control light transmission direction by switching the magnetization direction of the GHM slab.

A GHM is based on a hyperbolic metamaterial, which is generally a bilayer superlattice consisting of a metal layer and a dielectric layer stacked along the normal 

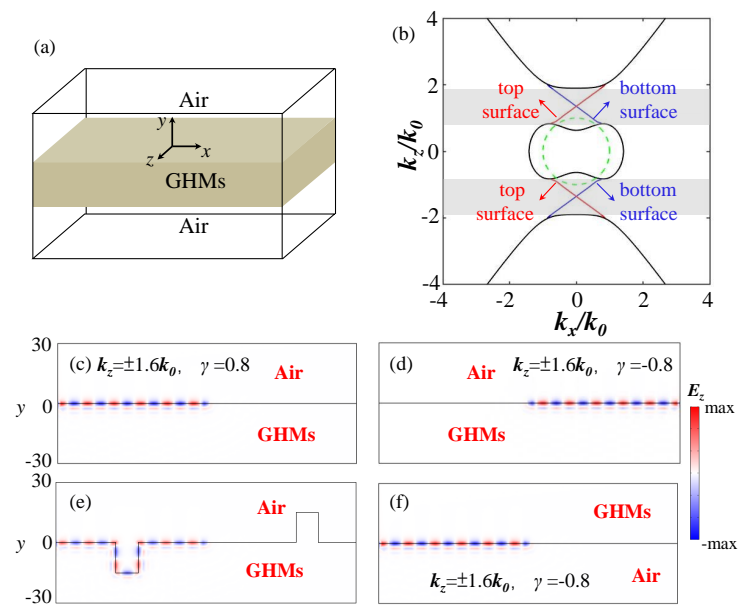

FIG. 4. One-way propagating edge states. (a) A GHM slab in air. (b) Calculated EFS dispersions for the GHM slab in air (a). Black, red, and blue lines denote dispersions of bulk state, top and bottom edge states, respectively. The dashed green circle denotes light cone. (c) and (d) Simulated propagations of light emitted by a line source at $x=0$ on the top surface at $k_{z}=1.6 k_{0}$ with $\gamma=-0.8$ and $\gamma=+0.8$, respectively. (e) The same as in (c) except that the surface is now uneven, which does not stop light propagate along the negative $x$-direction. (f) The same as in (d) but on the bottom surface.
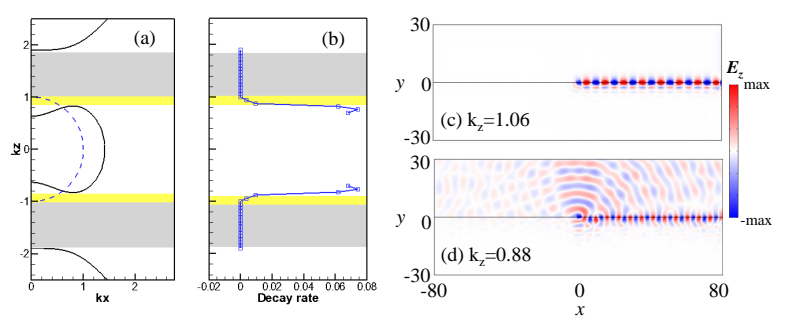

FIG. 5. (a) EFS dispersions and (b) decay rate of the electromagnetic waves for the GHM slab in the air. In (a), the solid black and dashed blue curves denote the dispersions of the bulk states and the air, respectively. In (a) and (b), the yellow regions denote the overlap regions of the air dispersion and bulk gap regions, while the grey regions denote the complete gap regions. (c) and (d) Electric field distributions of the edge states with $k_{z}=1.06$ and $k_{z}=0.88$, respectively. Note that in (c), the electric field is completely confined to the edge of the GHM slab and hence there is no radiative energy loss, because the $k_{z}$ is outside the light EPS sphere. In contrast, in (d), because the $k_{z}$ is within the light EPS sphere, the electromagnetic wave of the edge state propagates strongly into the air, resulting in a large radiative energy loss. direction (see next Section for details). Thus, the GHMs possess the property of surface plasmon polaritons (SPPs). Compared with the electromagnetic waves in the air, the SPPs are slower waves and hence have larger wave vectors than that of light in the air. As a result, the edge states on a GHM may inherit this larger wave vector propagation, as shown in Fig. 5. Therefore, there may be no intersection between the air light line and edge states, i.e., the edge states are out of the light line. Consequently, the edge state would propagate on the interface without radiation [Fig. 5(c)] because the edge states cannot couple to the electromagnetic waves in the air. In strong contrast, most of photonic topological insulators are made of photonic crystals and consequently, the one way edge states on the interfaces of these insulators suffer from severe radiation loss and a metal wall serving as a cladding perfect electric conductor or a perfect magnetic conductor has to be added to the surrounding edges to stop radiation loss into the air ${ }^{22}$. In practice, there is no lossless perfect conductor and even good metals such as copper used as the cladding walls absorb the radiation significantly. This certainly hinders their applications. In contrast, in a GHM, the air serves as the good insulator for stopping the radiation of the edge states into the air [Fig. 5(c)].

\section{NEGATIVE REFRACTION OF EDGE MODE}

Another interesting property of the topological surface states of the GHM is negative refraction, similar to that of the CHM reported recently in Ref. 35. As shown in Fig. 6 (b), the $x$ component of the group velocity (energy flow) of all the surface states of the GHM with $\gamma=-0.8$ (the blue line in the bottom panel) is positive $\left(v_{v, x}>0\right)$, while the phase velocity $\left(v_{p h, x}=\omega / k_{x}\right)$ changes sign across $k_{z}=0$. As a result, in the $k_{x}<0$ region the $x$ components of the group velocity and phase velocity have opposite signs $\left(v_{g, x}>0\right.$ and $\left.v_{p h, x}<0\right)$ [see Fig. 4(b)]. This is the signature of negative refraction $30 \mid 35$. On the contrary, the surface states on the GHM with $\gamma=0.8$ in the $k_{x}<0$ region have $v_{g, x}<0$ and $v_{p h, x}<0$ [see upper panel of Fig. 6(b)]. Following the method proposed in Ref. [36, we show in Fig. 6 that by drawing a vertical line in the EFS diagram (i.e., conservation of the momentum parallel to the interface), the group velocities of the three points intersecting the surface modes will give the propagation directions of the surface waves [Fig. 6(b)]. Consequently, as shown in Fig. 6(c), the incident (wave 1) and refracted (wave 3) surface waves lie on the same side of the normal line, indicating a negative refraction. The existence of negative refraction of the topological surface states would lead to many inter-

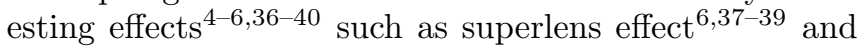
suppression of reflected waves 36 . 

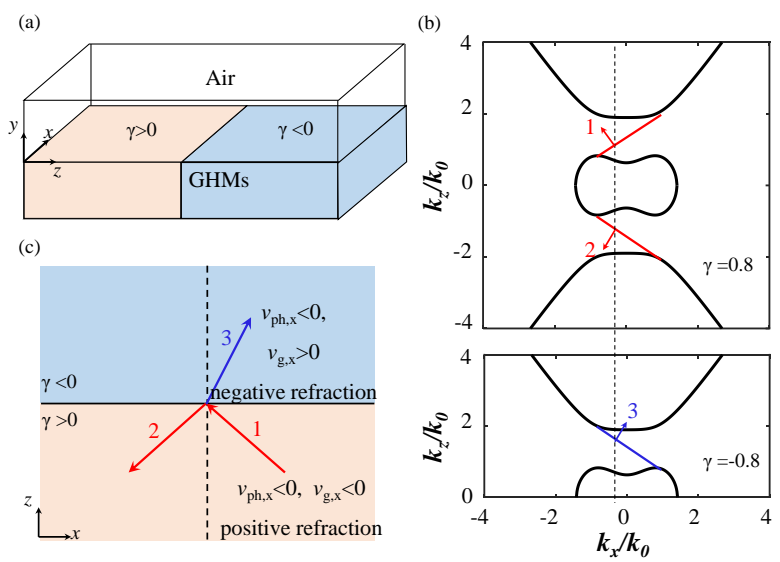

FIG. 6. Schematic illustration of the negative refraction of the TPSS of the GHM. (a) Side view and (b) the EFS of the system. (c) The incident, reflected, and transmitted waves at the interface between the two different GHM insulators. whose group velocities are denoted as 1, 2, and 3 in (b), respectively. Here $v_{v g, x}$ and $v_{p h, x}$ denote the $x$ components of the group and phase velocities, respectively.

\section{REALIZATION OF THE PROPOSED GYROMAGNETIC HYPERBOLIC METAMATERIALS}

The hyperbolic metamaterials are a highly anisotropic material with real parts of the principal components of its permittivity tensor having opposite signs 30 and have been intensively investigated because of this unique property. Here we consider a bilayer superlattice composed of a metal layer with relative permittivity $\epsilon_{m}$ and thickness $d_{m}$ and a dielectric layer with relative permittivity $\epsilon_{d}$ and thickness $d_{d}$ stacked along the $z$-direction. The effective dielectric constant of this metamaterial is given by

$$
\epsilon_{e f f}=\left[\begin{array}{ccc}
\epsilon_{x-y} & 0 & 0 \\
0 & \epsilon_{x-y} & 0 \\
0 & 0 & \epsilon_{z}
\end{array}\right]
$$

where

$$
\begin{aligned}
& \epsilon_{x-y}=\frac{\epsilon_{m} d_{m}+\epsilon_{d} d_{d}}{d_{m}+d_{d}}, \\
& \frac{1}{\epsilon_{z}}=\frac{d_{m} / \epsilon_{m}+d_{d} / \epsilon_{d}}{d_{m}+d_{d}} .
\end{aligned}
$$

If the dielectric layer is replaced by a gyromagnetic medium layer, the superlattice becomes a gyromagnetic hyperbolic metamaterial with its relative permeability tensors $\left[\begin{array}{ccc}\mu_{g} & -i \gamma & 0 \\ i \gamma & \mu_{g} & 0 \\ 0 & 0 & \mu_{g}\end{array}\right]$ and $\left[\begin{array}{lll}1 & 0 & 0 \\ 0 & 1 & 0 \\ 0 & 0 & 1\end{array}\right]$ for the gyromagnetic and metal layers, respectively. The bilayer superlattice with relative permittivity $\epsilon_{g}=\epsilon_{d}$ and thickness

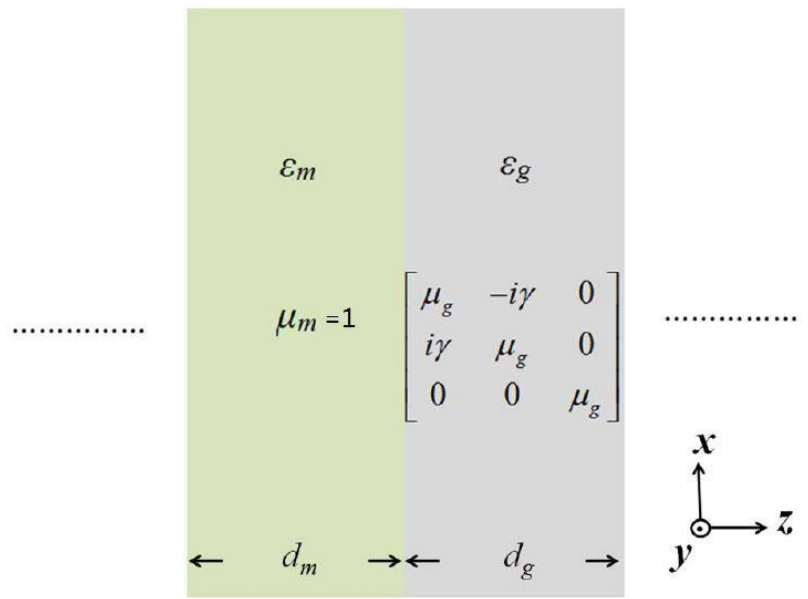

FIG. 7. Proposed gyromagnetic hyperbolic metamaterial as a bilayer superlattice composed of a gyromagnetic slab and a metal slab.

$d_{g}=d_{d}$ for the gyromagnetic layer, is schematically shown in Fig. 7.

For simplicity, let us write the fields in the metal as $\left(\mathbf{E}^{g}, \mathbf{H}^{g}\right)=\left(E_{x}^{g}, E_{y}^{g}, E_{z}^{g}, H_{x}^{g}, H_{y}^{g}, H_{z}^{g}\right)$ and in the gyromagnetic medium as $\left(\mathbf{E}^{m}, \mathbf{H}^{m}\right)=$ $\left(E_{x}^{m}, E_{y}^{m}, E_{z}^{m}, H_{x}^{m}, H_{y}^{m}, H_{z}^{m}\right)$. We assume that the wavelength is much larger than the thickness. Using the boundary conditions that $D_{z}, E_{x}, E_{y}, B_{z}, H_{x}$ and $H_{y}$ must be continuous across an interface, we obtain $\epsilon_{m} E_{z}^{m}=\epsilon_{g} E_{z}^{g}, E_{x}^{m}=E_{x}^{g}=E_{x}, E_{y}^{m}=E_{y}^{g}=E_{y}$, $H_{z}^{m}=\mu_{g} H_{z}^{g}, H_{x}^{m}=H_{x}^{g}=H_{x}, H_{y}^{m}=H_{y}^{g}=H_{y}$. Consequently, the average $D$-field and $B$-field are

$$
\begin{gathered}
D_{x}^{e f f}=\frac{d_{g} \epsilon_{g}+d_{m} \epsilon_{m}}{d_{g}+d_{m}} E_{x} \\
D_{y}^{e f f}=\frac{d_{g} \epsilon_{g}+d_{m} \epsilon_{m}}{d_{g}+d_{m}} E_{y} \\
D_{z}^{e f f}=\epsilon_{m} E_{z}^{m}=\epsilon_{g} E_{z}^{m} \\
B_{x}^{e f f}=\frac{d_{g} \mu_{g}+d_{m}}{d_{g}+d_{m}} H_{x}-\frac{i d_{g} \gamma}{d_{g}+d_{m}} H_{y} \\
B_{y}^{e f f}=\frac{d_{g} \mu_{g}+d_{m}}{d_{g}+d_{m}} H_{y}+\frac{i d_{g} \gamma}{d_{g}+d_{m}} H_{x} \\
B_{z}^{e f f}=\mu_{0} H_{z}^{m}=\mu_{g} H_{z}^{m} .
\end{gathered}
$$

Therefore, the effective relative permittivity and permeability tensors are given by

$$
\epsilon_{\text {eff }}=\left[\begin{array}{ccc}
\frac{d_{g} \epsilon_{g}+d_{m} \epsilon_{m}}{d_{g}+d_{m}} & 0 & 0 \\
0 & \frac{d_{g} \epsilon_{g}+d_{m} \epsilon_{m}}{d_{g}+d_{m}} & 0 \\
0 & 0 & \frac{\left(d_{g}+d_{m}\right) \epsilon_{g} \epsilon_{m}}{d_{g} \epsilon_{m}+d_{m} \epsilon_{g}}
\end{array}\right],
$$




$$
\mu_{e f f}=\left[\begin{array}{ccc}
\frac{d_{g} \mu_{g}+d_{m}}{d_{g}+d_{m}} & \frac{-i d_{g} \gamma}{d_{g}+d_{m}} & 0 \\
\frac{i d_{g} \gamma}{d_{g}+d_{m}} & \frac{d_{g} \mu_{g}+d_{m}}{d_{g}+d_{m}} & 0 \\
0 & 0 & \frac{\left(d_{g}+d_{m}\right) \mu_{g}}{d_{g} \epsilon_{m}+d_{m} \mu_{g}}
\end{array}\right]
$$

We can easily find suitable materials as the gyromagnetic medium and the metal slab to construct a GHM described above. For example, yttrium iron garnet (YIG) under an applied magnetic field of 1600 Gauss has the effective relative permittivity $\epsilon_{g}=15$ and permeability $\mu_{g}=1.12$ and $\gamma=0.124$ at $1.94 \mathrm{THz} \frac{15}{}$, and thus can be used as the gyromagnetic medium. InSb has the effective relative permittivity $\epsilon_{m}=-10.78$ at $1.94 \mathrm{THz} z^{\sqrt{11}}$ and thus can serve as the metal slab. In the bilayer multilayer, the thicknesses of the gyromagnetic medium and metal layers are taken to be the same and much less than 1.546x $10^{-4} \mathrm{~m}$ (wavelength of $1.94 \mathrm{THz}$ ). In this case, the effective relative permittivity and permeability tensors can be written as

$$
\begin{gathered}
\epsilon_{\text {eff }}=\left[\begin{array}{ccc}
2.11 & 0 & 0 \\
0 & 2.11 & 0 \\
0 & 0 & -76.73
\end{array}\right], \\
\mu_{\text {eff }}=\left[\begin{array}{ccc}
1.06 & 0.062 i & 0 \\
-0.062 i & 1.06 & 0 \\
0 & 0 & 1.06
\end{array}\right] .
\end{gathered}
$$

The EFS dispersions obtained using the effective parameters are also displayed in Fig. 8. Clearly, the shape of the EFS dispersions is similar to that of the EFS presented in the last section, and furthermore, the topological properties such as the Chern numbers of the bands and the topological nature of the band gaps are identical to those shown in the last section.

To see the robustness of the topological band gap against the variations of the material parameters, we show the calculated band gap as a function of both $\gamma$ and $\epsilon_{z z} / \epsilon_{x x}$ in Fig. 9. Clearly, the band gap is significant $(>0.5)$ in a wide range of the parameters. For example, when $\epsilon_{z z} / \epsilon_{x x}$ is in between -0.1 and -1.0, the band gap is significant for $\gamma$ being from 0.4 to 2.0. Interestingly, Fig. 9 shows that for $\epsilon_{z z} / \epsilon_{x x}$ is in between -1.0 and -2.0, one tunes the system from the gapless to the Chern insulating state and then back to gapless state. Finally, it should be pointed out that the present photonic Chern insulator would be much simpler to design and fabricate than many other photonic insulators. For example, the photonic Floquet topological insulators reported in Ref. [32] are composed of an array of micrometer-scale helical waveguides. The photonic chiral hyperbolic topological insulators proposed in Ref. [29] would be based on an array of hyperbolic coils which serve as chiral resonators. In contrast, as discussed above, the photonic Chern insulators would consist of a simple YIG/InSb bilayer superlattice, and thus would be much easier to fabricate.

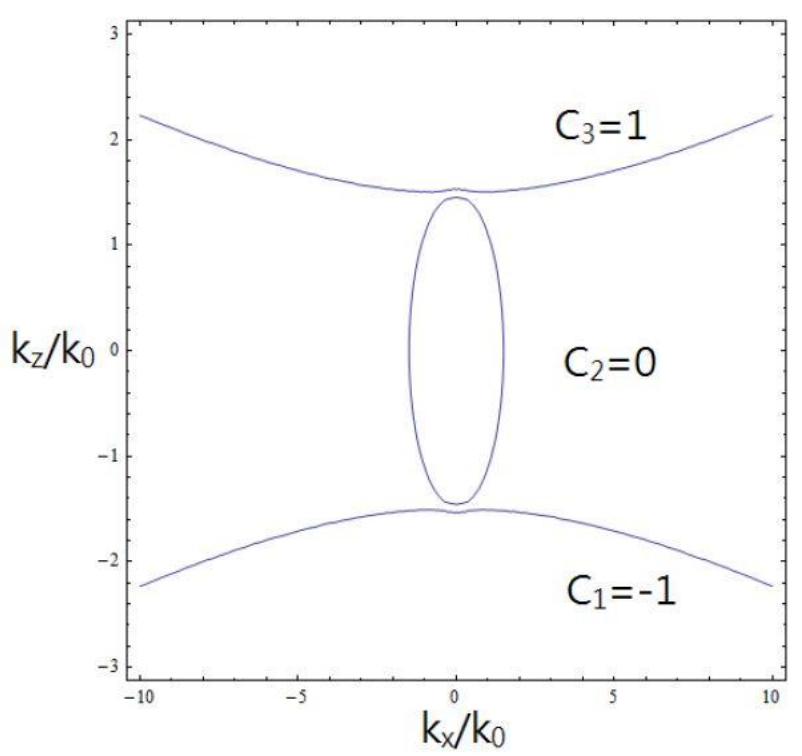

FIG. 8. Calculated EFS of the bilayer superlattice shown in Fig. 7 made of a YIG slab as the gyromagnetic medium and an InSb layer as the metal slab.

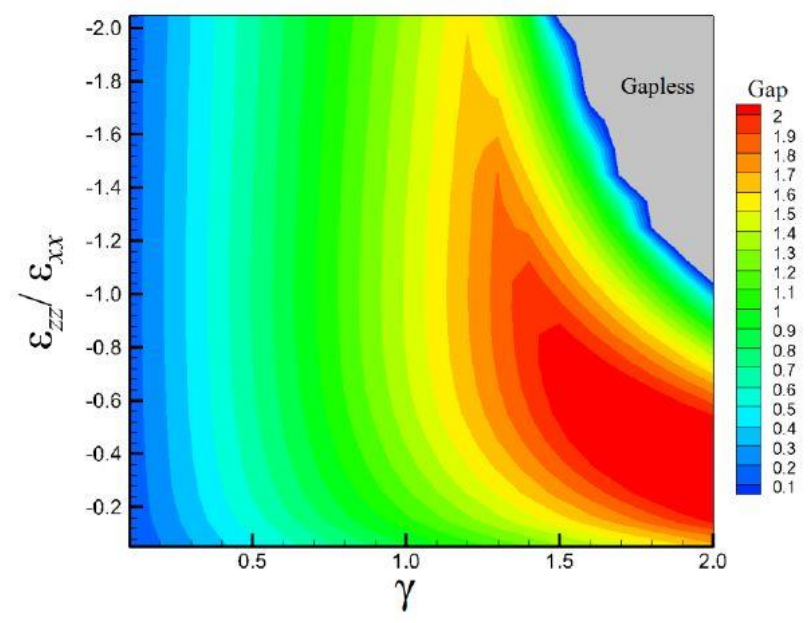

FIG. 9. Calculated band gap map on the $\gamma-\left(\epsilon_{z z} / \epsilon_{x x}\right)$ plane of the bilayer superlattice shown in Fig. 7, which is made of a YIG slab as the gyromagnetic medium and an InSb layer as the metal slab.

\section{DISCUSSION AND CONCLUSIONS}

In the QAH effect, vacuum is an insulator for electrons and thus the chiral edge states are localized at the surface of the Chern insulator. In contrast, vacuum to photons is often like a free electron metal and thus light propagation on the unidirectional edge modes would generally suffer from radiation loss. Consequently, a metal film such as copper is usually inserted between air and the topological photonic insulator to suppress the radiation leakage $e^{22}$. However, metals like copper could incur significant Ohmic loss. In strong contrast, the chiral 
edge modes in our GMHs are not only reflection-free but also non-radiative, as demonstrated by our finite element electromagnetic simulations (see Figs. 4 and 5). This is simply because the chiral edge dispersions are located outside light cone [see Fig. 4(b)]. This is an important advantage of the GMHs over the gyromagnetic photonic crystals 22223 .

In conclusion, by both theoretical analysis and electromagnetic simulations, we have demonstrated that gyromagnetic hyperbolic metamaterials (GHM) are photonic Chern insulators with fascinating properties. We further show that the large topogical band gaps with gap Chern number of one in these metamaterials, result from the simultaneous presence of the hyperbolicity and also the gyromagnetic effect, which breaks the time-reversal symmetry and thus gives rise to nonzero Berry curvatures on the EFSs. Remarkably, unlike many other photonic Chern insulators, the GHM Chern insulators possess non-radiative chiral edge modes on their surfaces, and thus allow to fabricate unidirectional waveguides without cladding metals which generally incurr considerable Ohmic loss. Furthermore, the photonic edge states in the proposed Chern insulators are robust against disorder on a wide range of length scales, in strong contrast to crystalline topological insulators, and the light flow direction on the surface of the Chern insulators can be easily flipped by switching the direction of an applied magnetic field. We also uncover negative refraction of the topological surface wave at the boundary between the GHMs with the opposite signs of gyromagnetic parameters. Finally, we show that compared with other photonic topological materials such as chiral hyperbolic materials ${ }^{29}$, the present GHM Chern insulators can be much easier to fabricate.

\section{ACKNOWLEDGMENTS}

This work is supported by the Ministry of Science and Technology, the National Center for Theoretical Sciences and the Thematic Research Program (AS-TP-106-M07) of the Academia Sinica of the R. O. C. (Taiwan).
* gyguo@phys.ntu.edu.tw

1 S. John, Strong localization of photons in certain disordered dielectric superlattices, Phys. Rev. Lett. 58, 2486 (1987).

2 E. Yablonovitch, Inhibited Spontaneous Emission in SolidState Physics and Electronics, Phys. Rev. Lett. 58, 2059 (1987).

3 D. R. Smith, J. B. Pendry and M. C. K. Wiltshire, Science 305, 788 (2004).

${ }^{4}$ V. G. Veselago, The electrodynamics of substances with simultaneously negative values of $\varepsilon$ and $\mu$, Sov. Phys. Usp. 10, 509 (1968).

5 H.-C. Chan, S. Sun, and G.-Y. Guo, Near-infrared lefthanded metamaterials made of arrays of upright split-ring pairs, J. Phys. D: Appl. Phys. 51, 265103 (2018).

6 J. B. Pendry, Negative refraction Makes a Perfect Lens, Phys. Rev. Lett. 85, 3966 (2000).

7 V. Klimov, S. Sun, and G.-Y. Guo, Coherent perfect nanoabsorbers based on negative refraction, Opt. Express 20, 13071 (2012).

8 L. Lu, J. D. Joannopoulos, and M. Soljačić, Topological photonics, Nat. Photonics 8, 821 (2014).

9 M. Z. Hasan and C. L. Kane, Colloquium: topological insulators, Rev. Mod. Phys. 82, 3045 (2010).

10 X.-L. Qi and S.-C. Zhang, Topological insulators and superconductors, Rev. Mod. Phys. 83, 1057 (2011).

11 H. Weng, R. Yu, X. Hu, X. Dai and Z. Fang, Quantum anomalous Hall effect and related topological electronic states, Adv. Phys. 3, 227 (2015).

12 F. D. M. Haldane, Model for a Quantum Hall Effect without Landau Levels: Condensed-Matter Realization of the "Parity Anomaly", Phys. Rev. Lett. 61, 2015 (1988).

13 C.-Z. Chang, J. Zhang, X. Feng, J. Shen, Z. Zhang, M. Guo, K. Li, Y. Ou, P. Wei, L.-L. Wang, Z.-Q. Ji, Y. Feng, S. Ji, X. Chen, J. Jia, X. Dai, Z. Fang, S.-C. Zhang, K.
He, Y. Wang, L. Lu, X.-C. Ma, and Q.-K. Xue, Experimental observation of the quantum anomalous Hall effect in a magnetic topological insulator, Science 340, 167 (2013).

14 F. D. M. Haldane and S. Raghu, Possible Realization of Directional Optical Waveguides in Photonic Crystals with Broken Time-Reversal Symmetry, Phys. Rev. Lett. 100, 013904 (2008).

15 Z. Wang, Y. D. Chong, J. D. Joannopoulos, and M. Soljačić, Reflection-Free One-Way Edge Modes in a Gyromagnetic Photonic Crystal, Phys. Rev. Lett. 100, 013905 (2008).

16 T. Ochiai and M. Onoda, Photonic analog of graphene model and its extension: Dirac cone, symmetry, and edge states, Phys. Rev. B 80, 155103 (2009).

17 Y. Poo, R. X. Wu, Z. Lin, Y. Yang, and C. T. Chan, Experimental Realization of Self-Guiding Unidirectional Electromagnetic Edge States, Phys. Rev. Lett. 106, 093903 (2011).

18 W. Tan, L. Chen, X. Ji, and H. Q. Lin, Photonic simulation of topological superconductor edge state and zero-energy mode at a vortex, Sci. Rep. 4, 7381 (2014).

19 S. A. Skirlo, L. Lu, and M. Soljačić, Multimode One-Way Waveguides of Large Chern Numbers, Phys. Rev. Lett. 113, 113904 (2014).

${ }^{20}$ W. He and C. T. Chan, The emergence of Dirac points in photonic crystals with mirror symmetry, Sci. Rep. 5, 8186 (2015).

21 H.-C. Chan and G.-Y. Guo, Tuning topological phase transitions in hexagonal photonic lattices made of triangular rods, Phys. Rev. B 97, 045422 (2018).

22 Z. Wang, Y. Chong, J. D. Joannopoulos, and M. Soljačić, Observation of unidirectional backscatteringimmune topological electromagnetic states, Nature 461, 772 (2009).

${ }^{23}$ Y. Yang, Y. Poo, R. X. Wu, Y. Gu and P. Chen, Ex- 
perimental demonstration of one-way slow wave in waveguide involving gyromagnetic photonic crystals, Appl. Phys. Lett. 102, 231113 (2013).

24 S. A. Skirlo, L. Lu, Y. Igarashi, Q. Yan, J. Joannopoulos, and M. Soljačić, Experimental Observation of Large Chern Numbers in Photonic Crystals, Phys. Rev. Lett. 115, 253901 (2015).

25 C. He, X.-C. Sun, X.-P. Liu, M.-H. Lu, Y. Chen, L. Feng, and Y.-F. Chen, Photonic topological insulator with broken time-reversal symmetry, Proc. Natl. Acad. Sci. 113, 4924 (2016).

26 A. B. Khanikaev, S. Hossein Mousavi, W.-K. Tse, M. Kargarian, A. H. MacDonald, and G. Shvets, Photonic topological insulators, Nat. Mater. 12, 233 (2013).

27 T. Ma, A. B. Khanikaev, S. H. Mousavi, and G. Shvets, Guiding Electromagnetic Waves around Sharp Corners: Topologically Protected Photonic Transport in Metawaveguides, Phys. Rev. Lett. 114, 127401 (2015).

28 X. Cheng, C. Jouvaud, X. Ni, S. H. Mousavi, A. Z. Genack and A. B. Khanikaev, Robust reconfigurable electromagnetic pathways within a photonic topological insulator, Nat. Mater. 15, 542 (2016).

29 W. Gao, M. Lawrence, B. Yang, F. Liu, F. Fang, B. Beri, J. Li, and S. Zhang, Topological Photonic Phase in Chiral Hyperbolic Metamaterials, Phys. Rev. Lett. 114, 037402 (2015).

30 A. Poddubny, I. Iorsh, P. Belov, and Y. Kivshar, Hyperbolic metamaterials, Nat. Photon. 7, 948 (2013).

31 M. G. Silveirinha, Chern invariants for continuous media, Phys. Rev. B 92, 125153 (2015).

32 M. C. Rechtsman, J. M. Zeuner, Y. Plotnik, Y. Lumer, D. Podolsky, F. Dreisow, S. Nolte, M. Segev and A. Szameit,
Photonic Floquet topological insulators, Nature (London) 496, 196 (2013).

${ }^{33}$ T. Fukui, Y. Hatsugai, and H. Suzuki, Chern Numbers in Discretized Brillouin Zone: Efficient Method of Computing (Spin) Hall Conductances, J. Phys. Soc. Jpn. 74, 1674 (2005).

34 M. I. Dyakonov, New type of electromagnetic wave propagating at the interface, Sov. Phys. JETP 94, 119123 (1988).

35 V. V. Klimov, D. V. Guzatov, I. V. Zabkov, H.-C. Chan, and G.-Y. Guo, Size and host-medium effects on topologically protected surface states in bianisotropic threedimensional optical waveguides, Phys. Rev. B 98, 075433 (2018).

${ }^{36}$ H. He, C. Qiu, L. Ye, X. Cai, X. Fan, M. Ke, F. Zhang, Z. Liu, Topological negative refraction of surface acoustic waves in a Weyl phononic crystal, Nature 560, 61 (2018).

37 N. Fang, H. Lee, C. Sun, and X. Zhang, Sub-diffractionlimited optical imaging with a silver superlens, Science 308, 534 (2005).

38 Z. Liu, H. Lee, Y. Xiong, C. Sun, and X. Zhang, Farfield optical hyperlens magnifying sub-diffraction-limited objects, Science 315, 1686 (2007).

39 I. I. Smolyaninov, Y.-J. Hung, and C. C. Davis, Magnifying superlens in the visible frequency range, Science 315, 1699 (2007).

40 K.-M. Lin and G. Y. Guo, Uncoupled modes and all-angle negative refraction in walled honeycomb photonic crystals, J. Opt. Soc. Am. B 25, C75 (2008).

${ }^{41} \mathrm{H} . \mathrm{H}$. Wu and Y. C. Lan, Magnetic lenses of surface magnetoplasmons in semiconductor-glass waveguide arrays, Appl. Phys. Expr. 7, 032203 (2014). 